REGARDS

SUR L'ECONOMIE ALLEMAND

BULLETIN ECONOMIQUE DU CRRAC
Regards sur l'économie allemande

Bulletin économique du CIRAC

$114 \mid 2014$

Varia

\title{
La politique de restructuration menée par la Sarre
}

\section{Hanspeter Georgi}

Traducteur : Isabelle Bourgeois

\section{OpenEdition}

\section{Journals}

Édition électronique

URL : http://journals.openedition.org/rea/4724

DOI : $10.4000 /$ rea.4724

ISSN : 1965-0787

Éditeur

CIRAC

Édition imprimée

Date de publication : 1 octobre 2014

Pagination : 17-28

ISSN : 1156-8992

Référence électronique

Hanspeter Georgi, «La politique de restructuration menée par la Sarre », Regards sur l'économie allemande [En ligne], 114 | octobre 2014, mis en ligne le 01 octobre 2016, consulté le 01 mai 2019. URL : http://journals.openedition.org/rea/4724 ; DOI : 10.4000/rea.4724 


\section{La politique de restructuration menée par la Sarre}

\section{Hanspeter Georgi}

La Sarre, région frontalière avec la France et le Luxembourg, avait construit son essor économique et sa compétitivité industrielle sur ses deux ressources naturelles: le charbon et l'acier. Mais ce Land, qui fait partie de la République fédérale depuis 1957 seulement, a subi de plein fouet à partir des années 1960 les effets sur l'activité et l'emploi des crises successives de ce secteur, tout comme ses régions voisines, d'ailleurs : la Lorraine, la Wallonie et le Luxembourg. Seul le Luxembourg a pu surmonter rapidement cette crise structurelle ; il est vrai que cet Etat indépendant avait pu mettre en œuvre une politique autonome et ciblée de reconversion.

En Sarre, la gigantesque destruction d'emplois industriels liée à ces mutations structurelles a pu être largement compensée par l'implantation de nouvelles entreprises, essentiellement dans l'automobile et la construction mécanique. II en est issu une nouvelle dynamique qui fait qu'aujourd'hui, la Sarre est redevenue un site industriel compétitif et bien inséré dans une économie mondiale globalisée. Cette reconversion qui cherche à optimiser le potentiel de compétitivité régional n'est toutefois pas achevée. Pour débrider totalement sa croissance, le Land doit encore réduire sa dette et consolider ses finances publiques. Enfin, pôle industriel qui en jouxte trois autres aux difficultés structurelles comparables mais ancrés dans un contexte politique national différent, la Sarre ni ses voisines ne parviendront isolément à développer une dynamique régionale porteuse. Seule une action concertée au sein d'une Grande Région européenne permettra de répondre durablement au changement.

\section{Crise du charbon et de l'acier, puis reconversion}

La Sarre a été rattachée en tant que Land à la République fédérale d'Allemagne

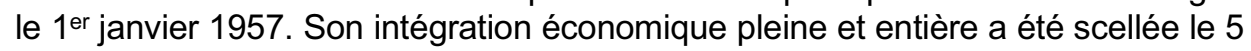
juillet 1959 en application de la Convention du règlement de la question sarroise signée entre la France et l'Allemagne le 2 juillet 1959. Onzième et dernier-né des Länder de la RFA d'avant l'Unité, la Sarre est aussi le plus petit, comptant un million d'habitants seulement. Et de par sa superficie $\left(2570 \mathrm{~km}^{2}\right)$, ce Land est comparable au Luxembourg voisin.

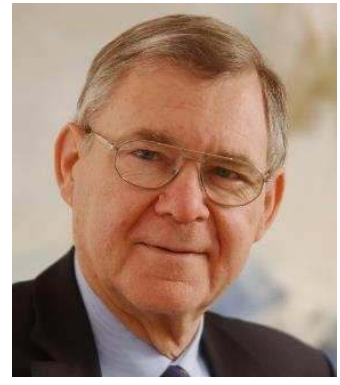

Dr. Hanspeter Georgi, chrétien-démocrate, ministre de 1'Economie du Land de Sarre de 1999 à 2007

La Sarre, dernier-né des Länder de l'ouest

Du fait de ses gisements de houille et de son industrie sidérurgique, cette région frontalière européenne a été l'objet de con-
flits entre la France et l'Allemagne tout au long de l'histoire (voir Behringer/Clemens, 2009). Ses racines françaises ont mar-
qué de leur empreinte l'économie sarroise comme en attestent la manufacture Villeroy\&Boch (fondée en 1748) ou le sidérur-
giste Dillinger Hütte (fondé en 1685 par le Marquis de Lenoncourt après autorisation de Louis XIV). Politiquement aussi, les
liens historiques sont restés vivaces. Deux grands noms restent associés à la ville de Saarlouis : celui de Vauban qui l'avait
érigée en place forte sur ordre de Louis XIV et celui du futur Maréchal Michel Ney (né en 1769).
Qui dit charbon et acier y associe aussi le nom de Robert Schuman - Lorrain, enfant de cette région transfrontières connue
sous le nom de SaarLorLux -, père de la Communauté du charbon et de l'acier et un des pionniers de l'actuelle Union euro-
péenne. On comprend dès lors le profond attachement des Sarrois à la fête de l'Europe, la célébration du 9 mai 1950.

Deux Länder en Allemagne de l'ouest ont subi de plein fouet l'impact des crises du charbon et de l'acier survenues depuis les années 1960 et achèvent aujourd'hui leur reconversion : la Rhénanie du Nord-Westphalie (région de la Ruhr) et la Sarre. Chaque Land étant souverain en matière de politique structurelle régionale (voir Bourgeois, 2007), les politiques menées concrètement diffèrent, certes, mais sont toutes basées sur le développement du potentiel existant. Dans le cas de la

\author{
Une économie \\ en reconversion \\ comme celle de la Ruhr
}


Perte d'emplois industriels au fil des crises du charbon et de l'acier...

... mais pas de désindustrialisation

Une vague de localisations industrielles...

... entraînant une réindustrialisation endogène
Sarre, les clés en sont notamment une longue tradition industrielle et une situation géographique favorable aux échanges transfrontières (SaarLorLux).

L'ampleur de la crise structurelle liée au charbon et à l'acier qu'a traversée la Sarre tient en peu de chiffres. Alors que voici une cinquantaine d'années, quelque 100000 Sarrois étaient occupés dans les secteurs du charbon $(60000)$ et de l'acier (40 000), aujourd'hui, seule la sidérurgie (et un seul groupe: Dillinger Hütte, Saarstahl AG) offre encore des emplois : 12000 au total. Le secteur minier quant à lui a cessé toute activité à la fin juin 2012, en application de la loi fédérale de 2007 sur le financement de la fin du subventionnement de l'extraction de la houille, adoptée après l'expiration du traité de la CECA en juillet 2002.

Mais cette perte d'emplois n'est pas synonyme de désindustrialisation. Car à la fin des années 1960 et au début des années 1970, quelque 120 nouvelles entreprises ont vu le jour, surtout dans les secteurs de l'industrie automobile et de la construction mécanique. Ces localisations exogènes répondant au besoin d'expansion d'entreprises existantes ont généré une dynamique de création endogène qui a développé l'activité dans les secteurs connexes.

Evolution du PIB sarrois de 1970 à 2012 (part des secteurs en \% du PIB)

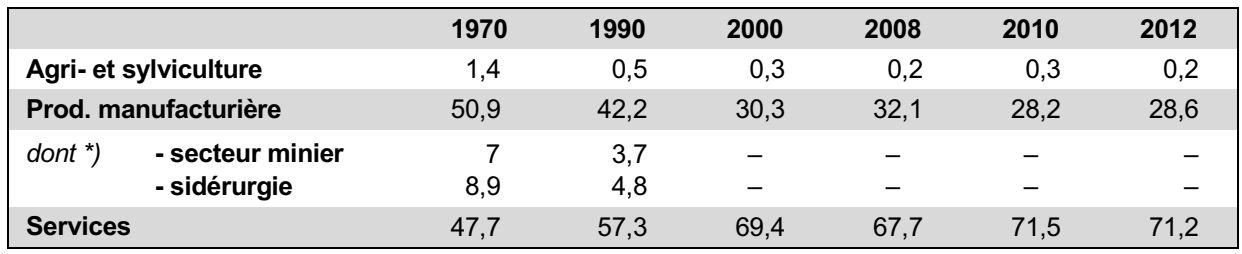

Source des données : Statistisches Amt Saarland. ${ }^{*}$ ) ces secteurs ne figurent plus dans les statistiques depuis 2000.

La première vague de localisations a été favorisée par une politique ciblée valorisant l'attractivité du territoire qui avait un atout maître : la disponibilité d'une main-d'œuvre qualifiée, partageant une culture et une expérience industrielles. Les pouvoirs publics du Land ont donc mené une politique offensive d'implantation de sites de production qui répondait à la demande de groupes industriels en quête d'expansion et d'ouverture de sites. Cette politique a été mise en œuvre notamment à l'aide d'un des outils du fédéralisme coopératif qu'est l'obligation faite à la Fédération de concourir financièrement à l'accomplissement des tâches des Länder si celles-ci ont une portée générale et si ce soutien est nécessaire à l'amélioration de la structure économique générale (voir REA 102/2011). Ce soutien fédéral à la politique de développement structurel régional de la Sarre a permis de soutenir financièrement l'investissement aboutissant à la création d'emplois. C'est ainsi que des entreprises comme Ford, ZF, Bosch, Eberspächer, Festo et bien d'autres encore ont ouvert de nouveaux sites de production en Sarre. Au fil du temps, ils se sont développés, se transformant en des "sites leaders » au sein des groupes présents : sites de production externalisés à l'origine, ils sont aujourd'hui des succursales à part entière en charge du développement produit de leur groupe (fonctions de management propres). Certaines de ces unités ont même développé des activités nouvelles, généralement complémentaires comme la distribution, la logistique et même la formation continue. C'est ainsi que nombre de ces unités de production installées en Sarre voici une cinquantaine d'années sont restées des acteurs majeurs de la compétitivité du Land, permettant à ce dernier de se placer parmi les dix grands sites de la filière automobile en Europe - et ce, en l'espace d'une génération seulement.

Parallèlement, la reconversion de l'économie sarroise et la création d'emplois ont été portées par le développement des échanges commerciaux et une réindustrialisation de type endogène. Cette dynamique est née elle aussi voici quelque 55 ans, avec la création d'entreprises industrielles à la réputation aujourd'hui mondiale comme le fabricant de matériel électrique Hager (dont l'un des sites de production se situe à Obernai) et le spécialiste de la gestion des fluides Hydac (avec entre autres un site à Forbach), ou encore le spécialiste de l'hydratation oculaire Ursapharm, le fabricant de compléments alimentaires et cosmétiques 
biologiques Theiss, le fabricant de pizzas surgelées Pizza Wagner, le brasseur Karlsberg ou, dans la distribution, le marchand de meubles Möbel Matin et l'importateur-distributeur de produits agroalimentaires Globus.

Industrie de la Sarre 1960-2013 : évolution des emplois par secteur *)

\begin{tabular}{|c|c|c|c|c|c|c|}
\hline & 1960 & 1980 & 2013 & 1960 & 1980 & 2013 \\
\hline & & en $\%$ & & \multicolumn{3}{|c|}{ nombre d'emplois } \\
\hline Extraction de houille et sidérurgie & 55,9 & 35,5 & 11,8 & 97828 & 54942 & 9875 \\
\hline Construction mécanique & 5,3 & 8,0 & 20,3 & 9314 & 12366 & 16975 \\
\hline Construction automobile & 0,5 & 11,7 & 23,7 & 927 & 18051 & 19804 \\
\hline Fonderies & 3,2 & 2,6 & 4,1 & 5609 & 3998 & 3418 \\
\hline Caoutchouc et plasturgie & 0,0 & 4,0 & 4,6 & 0 & 6180 & 3821 \\
\hline Autres & 22,5 & 23,6 & 24,9 & & & \\
\hline Total emplois industriels & 100 & 100 & 100 & 174897 & 154874 & 83703 \\
\hline
\end{tabular}

Source des données : Statistisches Amt Saarland, IHK Saarland. NB : 1960 et 1980 : entreprises > 20 salariés ; 2013 , > 50 salariés. *) Les contours des branches ou secteurs ayant varié, ces données ne sont que partiellement comparables entre elles.

Du fait de ces localisations tant exogènes qu'endogènes, la part de l'industrie dans l'économie de la Sarre est restée élevée, supérieure à la moyenne allemande $(25 \%)$. Quant à son taux d'exportation $(50 \%)$, il est comparable au niveau atteint par le Bade-Wurtemberg ou la Bavière, les deux grands Länder exportateurs allemands. Si on prend en considération également les exportations indirectes, c'est-à-dire les produits semi-finis destinés à la production des produits exportés, alors le taux d'exportation de l'industrie sarroise est le plus élevé en comparaison de tous les Länder allemands à en croire la $\mathrm{CCl}$ du Land. Pour donner un exemple concret : les équipements fabriqués en Sarre et assemblés à Munich font ainsi de la BMW série 5 un véhicule pour moitié sarrois. Fortement orientée vers l'export, l'économie sarroise, et donc le site Sarre, tirent ainsi profit de la mondialisation. Mais cette ouverture n'est pas sans risque, comme pour l'ensemble de l'économie allemande au demeurant: lorsque surviennent des crises mondiales comme celle de 2008, leurs répercussions se font immédiatement sentir et tassent l'activité. Mais quand la croissance est de retour, l'économie recouvre sa compétitivité. Autrement dit : les crises actuelles sont conjoncturelles et non structurelles comme ce fut le cas de celles du charbon et de l'acier.

\section{Les atouts qui ont favorisé la reconversion de l'économie sarroise}

Un des préalables au succès d'une telle reconversion économique - et même sa condition sine qua non - est l'effort consenti à la formation professionnelle en apprentissage (système dual). A l'issue de leur formation scolaire, et même après le baccalauréat, les apprentis sont formés dans quelque 350 métiers de l'industrie, du commerce ou des services. Ils passent deux à trois jours par semaine dans l'entreprise formatrice et un ou deux jours dans leur école professionnelle. Ainsi se trouvent non seulement étroitement conjuguées formation théorique et pratique, mais également posés les jalons de la capacité à innover qui caractérise les ressources humaines outre-Rhin (voir Lasserre, 2014).

En Sarre, les pouvoirs publics et les entreprises (ce sont les chambres consulaires qui supervisent la formation professionnelle au plan régional) ont toujours cherché - même en période conjoncturelle difficile - à mettre à disposition des jeunes en quête de formation professionnelle et disposant des qualifications requises une place d'apprentissage en entreprise. Du fait de la forte implication des milieux économiques, le système dual est très réactif : les profils des métiers sont en permanence ajustés à l'évolution du marché, voire redéfinis pour mieux répondre à la demande. Cette adaptation s'effectue en quelque sorte du bas vers le haut, le rôle central dans l'évolution du système dual revenant aux entreprises
Une économie aujourd'hui bien positionnée dans la mondialisation

La formation dans le système dual 
L'ouverture du politique à la concurrence dans le domaine de la formation

Une recherche multi-positionnée et mondialement réputée dont l'engagement dans la formation professionnelle est particulièrement prononcé depuis toujours. L'offre de formation étant ainsi pilotée par le marché, la qualification de la main-d'œuvre présente sur le site constitue de ce fait un facteur décisif de l'attractivité et de la compétitivité d'une économie régionale, qu'il s'agisse de la Sarre ou de tout autre Land (voir Lasserre, 2014).

Le gouvernement sarrois soutient activement les initiatives innovantes en matière de formation qui émanent des entreprises. C'est le cas par exemple des formations en alternance dispensées par l'établissement d'enseignement supérieur (Fachhochschule) Hochschule für Wirtschaft und Technik (HTW), reconnues par l'Etat et qui portent le nom d' « études supérieures coopératives » (kooperatives Studium). Le Land a soutenu de la même manière création et développement de deux académies professionnelles (Berufsakademien) de droit privé : l'Akademie der Saarwirtschaft qui propose aux bacheliers des études supérieures en alternance, et la Deutsche Hochschule für Prävention und Gesundheitsmanagement pour la formation des cadres en charge de la prévention et du management dans le secteur de la santé. Ce que montrent ces exemples, c'est que, bien que les universités soient des établissements de droit public et qu'elles relèvent donc de la propriété publique, le politique non seulement n'entrave pas l'innovation privée dans le domaine de la formation mais considère comme salutaire la concurrence qu'elle génère. Cette approche aussi a contribué au succès de la reconversion de l'économie sarroise car elle traduit la capacité du politique à accepter le changement et à innover - un prérequis pour surmonter les crises structurelles.

Jusqu'au milieu des années 1980, la Sarre n'avait que peu d'institutions vouées à la recherche : deux universités (l'Université de la Sarre, créée par la France en 1949, et la HTW), et un Institut Fraunhofer mondialement actif malgré sa petite taille à l'époque (le Fraunhofer Institut für zerstörungsfreie Prüfverfahren ; méthodes de diagnostic des matériaux). La priorité de tous les gouvernements sarrois a dès lors été de combler d'urgence ce retard par rapport aux autres Länder. Car des structures de recherche dynamiques génèrent innovation, investissements et créent de l'emploi dans la région, a fortiori dans une économie du savoir. Selon le chercheur américain Richard Florida en effet, les politiques régionales qui accordent une importance particulière aux « $3 \mathrm{~T}$ » que sont talents, technologies et tolérance, ont un net avantage compétitif dans la concurrence inter-régionale. Depuis le milieu des années 1980, la Sarre a donc consacré des efforts particuliers pour développer le tissu d'organismes de recherche fondamentale et appliquée en conjuguant mesures de politique économique régionale, soutien de la Fédération et fonds structurels de l'UE. Au fil du temps sont ainsi venus s'ajouter aux deux universités et à l'Institut Fraunhofer cités ci-dessus de nombreux établissements et organismes de recherche fondamentale et appliquée mondialement réputés.

\section{Etablissements et organismes de recherche fondamentale et appliquée sis en Sarre}

- Deutsches Forschungsinstitut für Künstliche Intelligenz (DFKI), leader mondial dans la recherche fondamentale et appliquée sur l'intelligence artificielle (il a développé par exemple les systèmes de navigation automobile), au sein duquel a été récemment intégré le centre de recherche informatique de l'Université de la Sarre, l'Institut für Wirtschaftsinformatik (IWI) ;

- Leibniz-Institut für Neue Materialien (INM), spécialiste des nouveaux matériaux et connu pour ses travaux dans le domaine des nanotechnologies sur la base desquels est née une série de start-ups et de spin-offs ;

- Max-Planck-Institut für Informatik (MPI) et Max-Planck-Institut für Softwaresysteme (MPS). Parmi les centres Max Planck, ce sont les deux seuls spécialistes dans ce domaine. Du fait de leur coopération étroite avec le DFKI et l'IWI, la Sarre est aujourd'hui l'un des sites en pointe dans la recherche informatique dans l'UE ;

- Leibniz-Zentrum für Informatik $\mathrm{GmbH}$ : plateforme d'échanges mondiale pour les spécialistes de l'informatique issus du monde de la recherche et du milieu des entreprises ;

- Fraunhofer-Institut für Biomedizinische Technik (IBMT). Ses travaux sur la cryotechnologie appliquée au stockage de cellules d'origine végétale, animale et humaine se sont attiré récemment une réputation mondiale (cette technologie joue un rôle clé dans le cadre du programme de lutte contre le SIDA mené à l'échelle mondiale par la Fondation Gates) ;

- Helmholtz-Institut für Pharmazeutische Forschung (HIPS). Ce centre de recherche bio-pharmacologique est issu du Stiftungsinstitut für pharmazeutische Biotechnologie né d'un partenariat public-privé ;

- Zentrum für Mechatronik und Automatisierungstechnik (ZEMA). Ce centre spécialisé dans la mécatronique et les technologies d'automatisation est né à l'initiative et avec le soutien des entreprises sarroises avec pour mission notamment d'assurer la relève dans les sciences de l'ingénieur. 
Si l'historique de leur création diffère sur de nombreux points, il n'en suit pas moins un schéma identique en ce qui concerne leur financement : aux moyens apportés par l'UE, le Bund et le Land s'ajoutent leurs fonds propres. Autrement dit: chacun de ces organismes mène en parallèle travaux de recherche fondamentale et de recherche appliquée et travaille sur projets en coopération étroite avec les entreprises, répondant à leur demande en matière de R\&D. Le rôle des pouvoirs publics du Land se limite à mettre à disposition à proximité de ces producteurs de savoir les terrains et locaux nécessaires à l'activité des entreprises désireuses de se rapprocher de ces infrastructures de R\&D.

\section{Les cinq axes de la politique de reconversion sarroise}

Depuis 2000, la politique économique menée par le Land de Sarre suit cinq axes pour optimiser le potentiel de l'économie régionale et surmonter les effets de la crise structurelle du charbon et de l'acier qui continuaient à grever sa compétitivité malgré une réindustrialisation réussie. L'objectif était aussi de faire progresser le Land dans le palmarès des 16 Länder. Cette politique a été couronnée de succès : le PIB nominal par tête est passé de $22493 €$ en 1999 à $31834 €$ en 2013, permettant à la Sarre de se hisser de la $8^{\mathrm{e}}$ à la $7^{\mathrm{e}}$ place parmi les Länder où elle surclasse aujourd'hui Berlin. Le nombre total des emplois s'est accru, passant de 492000 à 510000 ; celui des emplois soumis à cotisations sociales passant de 347014 à 363 086. Quant au taux de chômage, il est tombé de 10,8 \% en 1999 à $7,3 \%$ en 2013, permettant à la Sarre de progresser de la $9^{e}$ à la $7^{e}$ place parmi les Länder. En ce qui concerne le taux de chômage des moins de 25 ans, la Sarre a même pu grimper de la $9^{\mathrm{e}}$ place $(14,1 \%)$ à la $4 \mathrm{e}(7,2 \%)$.

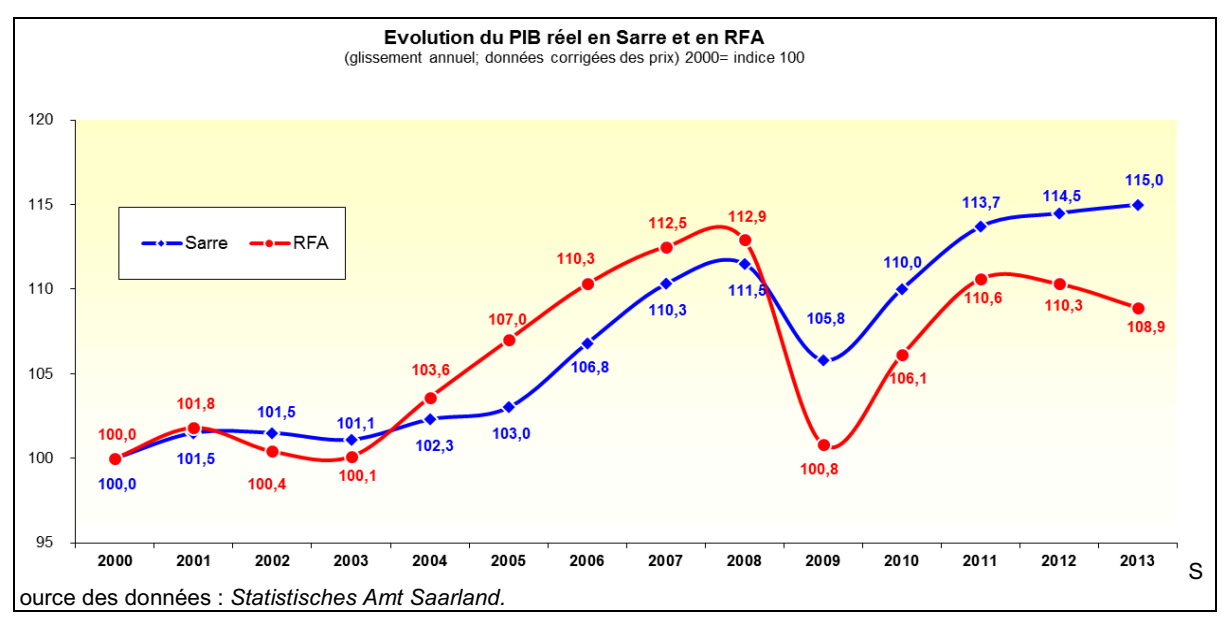

En comparaison, la Sarre a mieux réussi sa reconversion que la Ruhr (Rhénanie du Nord-Westphalie), l'autre grande région allemande aux prises avec la crise du charbon et de l'acier. Entre 1999 et 2011 par exemple, le nombre de chômeurs n'a baissé que de $10 \%$ dans la Ruhr, contre un peu plus de $30 \%$ en Sarre ; quant au nombre d'emplois soumis à cotisations sociales, la Ruhr les a vu reculer de $0,7 \%$ alors que la Sarre en a créé $3,2 \%$ de nouveaux. Et même en matière de hausse du revenu disponible par habitant, la Sarre surclasse la Ruhr, avec une hausse de $25,9 \%$ contre $18,4 \%$ seulement dans la Ruhr.

Une preuve a contrario de la bonne voie prise par la reconversion de l'économie sarroise est le fait que, en 2007, lors de la reconduction des aides à la restructuration versées au Land dans le cadre du programme fédéral de l'amélioration de la structure économie des Länder (Gemeinschaftsaufgaben) et du fonds EFRE de l'UE, le seuil pour le soutien aux investissements des grandes entreprises a été ramené à $20 \%$ (part de la population résidant dans une zone industrielle d'un seul tenant). A l'origine, il était certes question de supprimer ce programme de soutien, mais la bonne progression du PIB et de la création d'emplois ont plaidé

Une reconversion plus achevée que celle de la Ruhr 
Les cinq grands axes de politique économique

Axe 1 : promotion de l'esprit d'entreprise en faveur de la Sarre. Depuis 2007, Le Land ne dispose plus que de moyens modestes versés par le Bund au titre du programme de soutien 2007/14 à l'amélioration de la structure économique du Land (quelque 2,5 millions $€$ en moyenne annuelle). Quant aux fonds du programme EFRE 2007-2013/15 destinés au soutien à l'investissement créateur d'emplois dans l'industrie, le commerce et le tourisme, ils ne s'élèvent qu'à 17 millions $€$. Néanmoins, le Land dispose au cours de la période 2007-2014 d'un total de 126,5 millions $€$ pour soutenir le développement des infrastructures nécessaires à son économie.

La politique de développement économique que poursuit la Sarre depuis 2000 suit les cinq grands axes suivants : promotion de l'esprit d'entreprise, ouverture des pouvoirs publics et de l'administration aux impératifs des milieux économiques, volonté d'innover, approche positive de la restructuration de l'économie et stratégie offensive de valorisation du site.

Comme toutes les régions où prédomine la grande industrie, la Sarre présente par définition un taux très faible de travailleurs indépendants. Un changement de paradigme s'imposait donc pour la politique économique en ce qui concerne les créations d'entreprise. Les pouvoirs publics ont dès lors entrepris de créer un climat favorable à la création d'entreprises afin de diversifier la structure des entreprises dans le Land.

\section{Cette politique de promotion de l'esprit d'entreprise s'est traduite par les mesures suivantes :}

- création du réseau Saarland Offensive für Gründungen SOG. II associe par des liens de coopération étroits tous les acteurs impliqués dans la création d'entreprises: ministère de l'Economie de la Sarre, $\mathrm{CCl}$, chambre des métiers, banque publique Saarländische Investitions- und Kreditbank SIKB, Business Angel Saar BANS, etc. ;

- création dans tout le Land de nombreuses plateformes de créateurs d'entreprises et de PME, soutenues par le Land, afin de faciliter la création d'entreprises qui sont généralement le fait d'anciens salariés ;

- repositionnement de la banque publique de soutien aux entreprises SIKB sur le créneau des aides à la création d'entreprises ;

- transfert de technologies grâce à la création d'entreprises via le soutien aux start-ups et aux spin-offs ;

- création, pour la phase de démarrage, de pépinières auprès des universités et de deux ScienceParks pour la phase de développement des start-ups ;

- poursuite du développement des créations d'entreprise à l'Université de la Sarre où a été fondé un Campus de créateurs (Gründer Campus Saar). Ce projet d'université créatrice d'entreprises a été reconnu par la Fédération qui l'a doté d'importants moyens destinés à son développement ;

- soutien également aux activités privées au sein de ce Campus. Ainsi par exemple, le Prof. Scheer a été autorisé à y monter une pépinière (Scheer Group) qui accueille des spin-offs créées par des chercheurs en sciences de l'informatique. Une telle pratique reste encore l'exception en Allemagne..

Axe 2: pouvoirs publics et administration ouverts au monde de l'entreprise
La manière dont les pouvoirs publics et l'administration d'un Land ou d'une commune abordent les acteurs économiques est elle aussi déterminante pour réussir la reconversion d'un site. C'est ainsi que la société de promotion économique de la Sarre GWSaar peut s'enorgueillir de doubler en rapidité plus d'un Etat américain en ce qui concerne l'implantation d'entreprises. En effet, entre le moment où une entreprise décide de la localisation de son site et celui où elle démarre la production dans ses nouvelles installations, il ne s'écoule guère que six mois. Le slogan du "Land où les circuits sont rapides " ("Land der kurzen Wege ») n'a rien d'un vœu pieux, il est réalité vécue.

En Allemagne, le respect des intérêts des PME du Mittelstand a priorité.

La Sarre a mis en œuvre les mesures suivantes qui ont fortement contribué à accroître la compétitivité des siennes :

- repositionnement de la banque SIKB en prestataire de services aux entreprises (avec, par exemple, obligation de respecter certains délais pour traiter les demandes de crédit) ;

- regroupement de divers établissements bancaires publics (les 'banques maison' des PME) sous le toit de la SIKB de manière à ce que les PME disposent d'une sorte de guichet unique pour leurs besoins en financement ;

- création de nouveaux fonds de financement et de participation destinés aux PME ;

- stratégie active de promotion du commerce extérieur visant la prospection de nouveaux marchés grâce à des voyages d'études (souvent sous pilotage politique pour bénéficier de l'effet 'sésame ouvre-toi'), des plateformes de coopération, des stands individuels ou communs dans les foires-expositions et salons professionnels

Axe 3 : promotion de l'innovation...
Le transfert de technologies des infrastructures de recherche vers les entreprises emprunte trois voies: la création d'entreprises, les projets conjoints avec les entreprises et le transfert de chercheurs. L'exemple le plus célèbre dans l'Allemagne entière en ce qui concerne cette dernière voie est celui du Prof. Scheer, 
spécialiste de l'informatique appliquée aux entreprises à l'Université de la Sarre, qui a fondé la spin-off IDS Scheer et en a fait un global player aujourd'hui racheté par la société Software AG. Pour mettre plus efficacement en réseau le monde de la recherche et celui des entreprises à l'échelle de l'ensemble du Land, le gouvernement de la Sarre a lancé à partir de l'an 2000 plusieurs pôles de compétitivité (clusters) dans le cadre de sa stratégie d'innovation qui s'inspire des travaux du chercheur américain Michael Porter. Selon M. Porter, un cluster se définit par un ensemble de réseaux liant les divers acteurs économiques d'une région, à savoir les industriels, les distributeurs, les fournisseurs, les établissements de recherche, les prestataires de service, les entreprises artisanales, ainsi que leurs institutions que sont par exemple les $\mathrm{CCl}$ ou les fédérations professionnelles. Ces réseaux naissent au fil de l'intensification des échanges entre ces acteurs tout au long d'une chaîne de création de valeur et mènent à un grappage régional des activités favorisé par l'attractivité d'un site.

Conformément à cette définition ont ainsi été lancés en Sarre des clusters dans les secteurs suivants: automotive, NanoBioNet, technologies de l'information, logistique, énergie, technologies pharmaceutiques et médicales, économie de la santé. Leur mode de fonctionnement est identique et comprend d'une part le maillage des entreprises sarroises (business matching), mais également la coopération avec d'autres clusters régionaux ou supra-régionaux, d'autre part des voyages d'études communs et des stands conjoints lors des foires et salons professionnels, une coopération étroite avec les universités et les centres de recherche extra-universitaires, ainsi que la mise en réseau avec les institutions en charge de la formation professionnelle initiale et continue.

Parmi ces pôles de compétitivité, deux se sont particulièrement bien développés : automotive et NanoBioNet. La Sarre, avec ses quelque 50000 emplois dans l'industrie automobile, est en Europe l'un des sites leaders dans cette branche ; la constitution d'un cluster automotive s'imposait donc à l'évidence. Un des effets en a été que la Société Fraunhofer, en accord avec le gouvernement du Land, a créé sur son site de Sarrebruck une nouvelle division, dédiée aux nouveaux matériaux et produits destinés à l'industrie automobile. Un autre, le fait que, à l'initiative de la direction de ce cluster, l'Ecole supérieure spécialisée (Fachhochschule) de Sarrebruck a créé un nouveau master "Automotive Production Engineering " accueillant des salariés en formation continue. A relever également que, dès l'origine, la fédération allemande de l'automobile a joué un rôle actif dans le comité de pilotage de ce cluster.

Le développement du cluster NanoBioNet a suivi un cheminement quelque peu différent. Le Leibniz-Institut für neue Materialien (INM) étant spécialisé dans la recherche appliquée sur la chimie et les nouveaux matériaux, et vu le nombre important de start ups créées soit par des chercheurs de l'INM soit par d'autres, spécialistes en sciences pharmaceutiques et biologie, et issus des laboratoires de recherche universitaires ou extra-universitaires du Land, le gouvernement sarrois a décidé, dans le cadre de sa politique économique, de lancer un cluster sur la base de ce réseau existant. II a ainsi fondé NanoBioNet e.V., une association chargée de gérer ce pôle de compétitivité. Du fait de la concentration des activités induite dans ces secteurs, les profils de métiers d'assistants de laboratoire en chimie et biologie ont été élargis aux nanotechnologies, et certains lycées ont programmé une nouvelle matière dans leur enseignement : les nanotechnologies. Enfin, avec le soutien financier du Land, plusieurs laboratoires d'initiation ont été créés sur le campus de l'Université de la Sarre afin de permettre aux collégiens et lycéens de découvrir les filières des mathématiques et des sciences de l'ingénieur. Cette étroite et fructueuse coopération entre entreprises et centres de recherche dans le secteur des nanotechnologies sous le patronage actif du ministère de l'Economie de la Sarre a valu reconnaissance à ce cluster, surnommé depuis NanoValleySaar. Quant à l'association NanoBio Net e.V. qui pilote ce cluster, elle a fondé depuis la Fédération allemande des nanotechnologies (Deutscher Verband Nanotechnologie) dont le siège est à Sarrebruck. Et
... et constitution de clusters

Cluster automotive

Cluster NanoBioNet 
Une approche holistique de l'innovation

Axe 4 :

une approche positive de la reconversion

Le défi actuel : consolider les finances publiques deux entreprises sarroises se sont taillé une réputation mondiale : Nanogate AG (400 salariés) et Nano-X GmbH (50 salariés).

Une autre exemple illustrant la politique régionale de développement des pôles de compétitivité est l'initiative prise par les entreprises du Land de fonder un Centre de recherche sur la mécatronique et de la robotique (ZEMA) dont la mission est le transfert des technologies grâce aux formations en sciences de l'ingénieur qu'il propose dans le but d'assurer la relève dans l'industrie sarroise.

Toutes ces mesures et initiatives suivent une approche holistique et ont de ce fait entraîné un changement de mentalité dans le monde universitaire. Grâce ne serait-ce qu'à la création de ScienceParks, de laboratoires d'initiation ou au développement des coopérations avec les entreprises, les milieux académiques se sont ouverts au monde de l'entreprise et à la globalisation, créant ainsi une culture elle aussi favorable à la reconversion du site. C'est ainsi que les liens de confiance et de coopération étroite entre établissements de formation et entreprises au sein des clusters ont débouché sur la création quasi just in time ou just in sequence de nouvelles filières, de nouveaux enseignements ou de nouveaux profils de métiers. Sans une telle interpénétration, ces innovations auraient pris beaucoup plus de temps ou n'auraient jamais vu le jour.

Les trois axes présentés révèlent à eux seuls une sorte de changement de paradigme dans la manière dont le politique aborde les défis du changement structurel. Et ils démontrent que pour surmonter les crises structurelles, ce qui compte, c'est que les pouvoirs publics régionaux assument leurs responsabilités et leurs compétences propres pour définir de nouvelles orientations de politique économique et les mettre en œuvre. Sans entrer dans le détail, le défi à relever à la fin du millénaire était le suivant: préserver à tout prix une économie construite presque exclusivement sur l'extraction de la houille ou, à l'opposé, anticiper la fin programmée de cette activité et adapter la politique économique en conséquence. La Sarre a alors fait le choix d'adopter une attitude proactive du changement. Et tant les pouvoirs publics que l'administration se sont alors découvert une mission nouvelle, qu'ils se sont appliqués à mettre en œuvre : assurer la coordination des diverses initiatives, servir d'interface entre les divers acteurs et leur permettre de coopérer. Car c'est généralement le décloisonnement des différents univers et de leurs approches propres qui nourrit la dynamique de l'innovation. Cette stratégie offensive a été couronnée de succès, comme le révèle le bon positionnement actuel de l'économie de la Sarre et la bonne tenue de son marché de l'emploi malgré l'impact de la crise de la finance mondiale dans les années 2008 et suivantes.

Aujourd'hui, la Sarre achève sa restructuration économique et se situe, quels que soient les indicateurs, dans le premier tiers du palmarès des Länder. Un défi demeure néanmoins : la situation budgétaire difficile du Land et de ses communes. Avec une dette par habitant de $16077 €$ en 2012, la Sarre vient en $3^{\text {e position }}$ des Länder les plus endettés d'Allemagne (en tête : Berlin, avec $30155 €$, suivi de Brême, avec $18213 €$ ). Depuis 2012, la Sarre fait partie des quatre Länder que le Conseil de la Stabilité (Stabilitätsrat), une autorité de contrôle budgétaire interne à l'Allemagne, considère comme menacés d'un risque de crise budgétaire. Cette autorité, créée en 2010 pour veiller notamment au respect, par le Bund et les Länder, du Pacte de stabilité et de croissance comme de la « règle d'or » budgétaire inscrite dans la Loi fondamentale (voir Bourgeois, 2011), a principalement une mission de prévention et d'accompagnement des collectivités endettées grâce à la définition conjointe d'un programme de consolidation budgétaire. La Sarre bénéficie d'un tel programme de redressement qui prendra fin en 2020. II constitue, dans le domaine des finances publiques, un défi comparable à celui que fut l'affrontement de la reconversion économique du Land. C'est donc la même approche proactive d'acceptation du changement qui doit guider sa mise en œuvre par l'actuel gouvernement de grande coalition (CDU/SPD) de la Sarre, avec le soutien des experts du Conseil de la Stabilité. Concrètement, il 
s'agira donc de débrider la compétitivité économique du Land tout en réduisant les dépenses publiques disproportionnées par rapport aux recettes. Dans ce domaine aussi, un changement de paradigme s'impose : il convient de briser tous les tabous en matière de réorganisation des administrations publiques (government ou administration process reengineering) afin d'accroître leur efficience. Ce n'est qu'ainsi que les pouvoirs publics pourront reconquérir toute leur latitude d'action en matière d'investissement.

La situation géographique de la Sarre dans l'espace européen et son appartenance à la Grande Région Saar - Lor - Lux - Rhénanie-Palatinat - Wallonie ont pris une dimension stratégique après la chute du Rideau de Fer et l'accélération de l'intégration européenne dont a bénéficié le secteur de la logistique. La politique économique régionale a sciemment misé sur cette évolution pour favoriser les localisations d'entreprises sur le territoire. Pour ne donner qu'un exemple du succès de cette politique de développement du site logistique : la commune de Überherrn et sa voisine française Creutzwald ont considérablement gagné en attractivité depuis qu'elles sont reliées à l'autoroute A4 en France par la jonction des routes nationales B269/N33.

L'attractivité de la région s'est accrue du jour au lendemain avec l'ouverture de la liaison TGV/ICE Paris-Est-Allemagne du sud et de son tracé nord Paris-Sarrebruck-Mannheim-Francfort. On comprend mieux la 'révolution' que représente cette liaison si on se souvient que, pour des raisons de politique militaire liées à I'histoire européenne, la Sarre comme la Lorraine avaient longtemps été défavorisées sous l'angle des infrastructures les reliant aux grandes métropoles suprarégionales. Alors que les déficits en matière d'infrastructures routières ont pu être largement comblés, bien que tardivement, cette Grande Région qui, aux prises avec les mêmes aléas de l'histoire comme les mêmes crises du charbon et de l'acier, a dû attendre longtemps avant d'être correctement raccordée au réseau ferroviaire européen à grande vitesse. Pendant de longues années, pouvoirs politiques et milieux économiques de la Sarre et de la Lorraine, épaulés par de nombreux partenaires de part et d'autre du Rhin, avaient dû plaider pour un tel raccordement avant que Kohl et Mitterrand, en un geste d'autorité, signent le 22 mai 1992 l'Accord de La Rochelle qui crée cette liaison. Aujourd'hui, pour garantir durablement l'attractivité de cette région, Merkel et Hollande devraient faire acte de la même autorité pour faire respecter les dispositions concernant le service sur cette liaison que contient l'Accord de la Rochelle. Concrètement, il s'agit d'assurer la même qualité de desserte sur les deux embranchements de cette liaison à grande vitesse : l'axe sud via Strasbourg et l'axe nord via Sarrebruck.

Un autre dossier franco-allemand prioritaire est le développement du fret fluvial. A cet effet, les écluses sur la Moselle doivent être rénovées et équipées d'un deuxième bassin. La canalisation de la Moselle est un projet qui remonte à une cinquantaine d'années ; dans le contexte du règlement de la question sarroise vis-àvis de la France, l'Allemagne avait alors donné son accord à ces travaux.
Axe 5 :

une stratégie offensive de valorisation du site

L'importance des infrastructures de transport

\footnotetext{
Le Land de Sarre a en outre pris une série d'autres mesures pour accroître et mieux valoriser encore son attractivité :

- un masterplan zones constructibles industrielles,

- soutien au développement du réseau à haut débit (fibre optique), surtout dans les zones rurales,

- construction d'un parc logistique pour les équipementiers du constructeur Ford (conçu, construit et géré par la holding publique des sociétés de promotion économique du Land : la Wirtschaftsförderungsgesellschaft Strukturholding Saar, SHS),

- conception et mise en œuvre d'une stratégie de promotion du tourisme pour une région qui, bien que présentant d'immenses richesses naturelles, n'a jamais été perçue comme une région touristique. Cette politique menée conformément à la logique des clusters a rapidement porté ses fruits, dynamisant le secteur des activités liées au tourisme,

- réhabilitation et valorisation touristique du site sidérurgique Völklinger Hütte, entré en 1994 au patrimoine culturel mondial de I'UNESCO. Ce site-témoin de la culture industrielle accueille aujourd'hui expositions, concerts et autres manifestations.
}

Sous la ministre-présidente Annegret Kramp-Karrenbauer (CDU), l'actuel gouvernement du Land de Sarre a engagé un nouveau chantier de valorisation : la mise en œuvre d'une «Stratégie France » (Frankreichstrategie) dont l'objectif est de généraliser la maîtrise de trois langues en l'espace d'une génération, l'allemand étant la langue maternelle, le français celle du voisin et l'anglais la langue

\author{
Une \\ "Stratégie France » \\ novatrice
}


de communication mondiale. Cette compétence linguistique sera un atout supplémentaire s'ajoutant à la position géographique centrale et au réseau d'infrastructures bénéficiant non seulement à l'attractivité de la Sarre, mais à celle de l'ensemble de la Grande Région. Rappelons que la qualité du réseau d'infrastructures et le multilinguisme de la population sont les facteurs déterminants pour la localisation d'un site de production que citent les dirigeants des entreprises mondiales ayant leur siège dans la région ARA (Amsterdam, Anvers, Rotterdam).

Un récent sondage de la $\mathrm{CCl}$ de Sarre (IHK Saarland), mené sur l'importance accordée par les entreprises à divers facteurs d'attractivité du territoire, révèle leur grande satisfaction vis-à-vis de l'état, mais aussi leur désir de voir le politique s'emparer d'un certain nombre de dossiers considérés comme stratégiques. Par ordre décroissant, les dix facteurs prioritaires à améliorer dans l'environnement réservé aux activités sont : le prix de l'électricité (voir à ce sujet REA 113/2014), le barème de la taxe communale prélevée sur les activités industrielles et commerciales (voir à ce sujet Röhl, 2009), la qualité de la formation des sortants du système scolaire (dont un certain nombre ne répond pas aux exigences du système dual), la qualité du réseau des infrastructures routières, le coût de la collecte et du traitement des déchets, l'image de la Sarre, la qualité des transports en commun de proximité, l'offre de structures permettant d'accueillir les enfants l'après-midi, le raccordement au réseau à très haut débit, et la qualité de l'accueil que l'administration réserve aux entreprises. Si ces desiderata sont loin d'être spécifiques au Land de Sarre, il en est un qui est particulièrement révélateur du potentiel de compétitivité spécifique du Land: le raccordement de la Sarre aux grands réseaux d'infrastructures. II vient certes en dernière position sur la liste des 15 points présentée aux entrepreneurs dans ce sondage; en effet, moins de $20 \%$ seulement des sondés se montrent insatisfaits de la situation. Mais ils sont plus de $80 \%$ à accorder une importance prioritaire à cette question - autant qu'à la disponibilité de la main-d'œuvre dans la région.

\section{Seule la coopération transfrontière dans la Grande Région permettra d'achever la restructuration de l'économie sarroise}

Au cours des décennies écoulées, les partenaires de la Grande Région que sont la Lorraine, le Luxembourg, la Région wallone, la Rhénanie-Palatinat et la Sarre ont activement travaillé à leur interdépendance structurelle en construisant des liens étroits entre leurs institutions et organisations dans les domaines politique, administratif, scientifique, économique, dans ceux de la formation, de la culture, des milieux patronaux et plus généralement des acteurs de la société civile. Et aujourd'hui, si on en croit Wikipedia, "Grande Région » est le terme consacré pour désigner cette région européenne ; il succède à appellation SaarLorLux.

Une « Vision d'avenir 2020 »

Ses partenaires partagent une vision : constituer un espace économique et de vie d'un seul tenant. Déjà, les échanges interrégionaux sont intenses, qu'il s'agisse de la mobilité des travailleurs (120000 franchissent quotidiennement les frontières), des consommateurs, des touristes, des étudiants, des personnes en quête d'un logement, etc.. Dans leur action quotidienne, les pouvoirs publics suivent les idées directrices de la "Vision d'avenir 2020 » (« Zukunftsbild 2020 »), un guide élaboré et présenté en 2003 par une Commission politique composée de représentants des régions partenaires et présidée par Jacques Santer, ancien Président de la Commission européenne.

L'Allemagne, partenaire incontournable

La Grande Région commence à devenir réalité, mais beaucoup reste à faire. Les de la Lorraine pouvoirs publics et les acteurs économiques comme ceux de la société civile s'y emploient, convaincus que le potentiel de développement régional se potentialise bien mieux par une action de concert qu'isolément. Le Conseil Economique, Social et Environnemental de Lorraine (CESEL) illustre bien cette approche de levier de développement dans une étude publiée en 2011 : " L'Allemagne, partenaire incontournable de la Lorraine-Deutschland, unverzichtbarer Partner Lothringens ». 
Petit à petit émerge de la sorte une identité européenne s'ajoutant à chacune des identités régionales et nationales. Néanmoins, les choses iraient bien plus vite, et le cadre réservé aux activités des entreprises comme des salariés serait déjà bien plus porteur si toutes les régions partenaires disposaient de pouvoirs comparables pour mettre en œuvre cette politique. Mais du fait de ces disparités, les habitants de la Grande Région ont souvent l'impression que l'intégration progresse à la vitesse d'un escargot. Pourtant, les exemples de bonnes pratiques et même de réalisations couronnées de succès ne manquent pas.

En matière d'emploi, un observatoire interrégional du marché du travail et une task force travailleurs transfrontaliers ont a été mis en place ; diverses initiatives visant à ouvrir le système de formation duale par-delà les frontières se précisent. L'accord conclu entre le président de la Région Lorraine et la ministre-présidente du Land de Sarre jouent dans ce domaine un rôle pionnier.

En ce qui concerne l'enseignement, citons les accords conclus entre la Sarre et le Département de la Moselle pour que, dans les écoles primaires, la langue du voisin soit enseignée par des locuteurs natifs. Au niveau de la coopération universitaire, la réputation de la DFHI/ISFATES (coopération entre Sarrebruck et Metz) n'est plus à faire, ni non plus celle de l'Université franco-allemande. Enfin, pour promouvoir la mobilité des étudiants comme des enseignants-chercheurs au sein de la Grande Région, un campus propre est en cours de développement, grâce au soutien du programme Interreg de l'UE.

Dans le domaine du tourisme, un concept commun de marketing est à l'étude dans le cadre du programme Interreg; il fait suite au projet de création d'une agence de tourisme unique proposé par la commission "Vision d'avenir 2020 », et qui n'avait débouché sur aucune innovation en matière de coopération transfrontières. Or le secteur du tourisme démontre de manière exemplaire comment une coopération ciblée permettrait de créer de la valeur ajoutée. Ainsi, en Sarre se trouve le site sidérurgique Völklinger Hütte inscrit au patrimoine mondial et dont le rayonnement traverse les frontières. Dans la Moselle, à Petite Rosselle, se trouve le Musée Les Mineurs Wendel, autre joyau de l'histoire de la mine. Plutôt que de réserver à la Sarre l'exposition sur l'histoire des activités minières dans le Land, cessées en 2012 ( (Das Erbe »), ne serait-il pas plus logique de l'intégrer également au Musée Les Mineurs Wendel ? Une telle mutualisation valoriserait les deux sites (et les deux régions).

Une des plus belles réalisations de la coopération économique est l'ouverture du World Trade Center à Metz voici plus de vingt ans, un projet réalisé grâce à la concertation entre les $\mathrm{CCl}$ de Metz et de Sarrebruck et financé grâce aux fonds européens. En ce qui concerne la politique des clusters, il serait souhaitable que les responsables politiques ne se contentent pas de déclarations d'intention à propos de la coopération entre les pôles de part et d'autre de la frontière. Lors du sommet de la Grande Région en 2011, Jean-Pierre Masseret, Président de la Région Lorraine, avait fait de la coopération transfrontière des clusters une priorité de sa présidence de la Grande Région. Dans le même ordre d'idées, il conviendrait aujourd'hui d'approfondir la coopération dans le domaine de l'énergie, au moins au niveau bi- ou trilatéral. Mais dans ce domaine comme dans tant d'autres, la réalisation des projets est souvent mise en échec par la défense de prétendus intérêts nationaux avec pour conséquence que les régions situées au centre de l'Europe comme la Sarre ou la Lorraine sont condamnées à végéter aux confins des territoires nationaux alors que, en coopérant plus étroitement pour approfondir leur intégration, elles pourraient débrider leur potentiel de croissance, de compétitivité et d'emploi.

Si responsables politiques, administrations et société civile organisée posent ainsi les jalons d'une vision d'une Grande Région au sein de l'espace économique européen, le tempo de sa réalisation comme l'intensité de son intégration dépendent aussi de l'adhésion de ses citoyens au projet. II convient de saluer ici par exemple la création de deux clubs transfrontières par des associations de type 
Rotary. Ou, dans le même objectif d'impulser cette dynamique, la constitution d'une association citoyenne : I'Institut de la Grande Région (Institut der Großregion) qui, sous la présidence de Roger Cayzelle, Président du CESEL, travaille à l'actualisation de la « Vision d'avenir 2020 » et à la mise au point d'une enquête auprès d'un large public afin de dresser une liste de recommandations à destination des responsables politiques régionaux et nationaux respectifs.

PoURQUOI CE PORTRAIT DE LA GRANDE RÉGION ? LA SARRE, malgré une politique de reconversion et de développement régional conséquente et persévérante, ne parviendra à relever pleinement le défi du changement structurel que si le Land n'est plus confiné aux marges du territoire national, mais peut occuper sa place au centre de l'Europe. Le même constat vaut pour la Lorraine, la Wallonie, la Rhénanie-Palatinat et, à moyen terme, même pour le Luxembourg. Ces régionspartenaires ne pourront chacune développer et consolider leur propre compétitivité que grâce à la création d'un espace économique et d'un bassin d'emploi communs. Or l'ancrage de cette Grande Région dans la carte des territoires attractifs au sein de l'UE ne pourra se faire à moyen terme que grâce à une politique conjointe de développement structurel régional ; encore faut-il créer les infrastructures institutionnelles nécessaires. Les programmes structurels européens tels Interreg, EFRE ou FSE pourraient y contribuer utilement, à condition toutefois qu'ils comportent des mesures d'incitation à l'intégration institutionnelle.

A fortiori, dans la perspective de la restructuration des Régions en France, une union de cette région transfrontière SaarLorLux prend une dimension existentielle pour la Lorraine comme pour la Sarre. La confiance mutuelle créée par des décennies de coopération aux niveaux politique, économique, administratif, mais aussi dans l'éducation, la culture et dans la société civile, est une base solide et porteuse pour le saut qualitatif aujourd'hui incontournable vers plus d'intégration. Faute d'une telle avancée, ces régions perdront tous les avantages liés à leur situation de confluence entre trois Etats membres riverains.

Pour l'UE aussi, une intégration plus poussée des régions jouxtant ses frontières intérieures présente un intérêt majeur. Car la 'valeur ajoutée' de l'UE est multidimensionnelle : dans un marché commun naissent peu à peu une conscience, voire une identité européennes. L'intégration d'une Europe unie dans sa diversité se fera d'abord le long de ses frontières intérieures - ou elle échouera. L'européanisation de régions frontalières comme la Grande Région SaarLorLux sera porteuse de sens pour le processus d'intégration de l'UE dans son ensemble car la création d'un marché commun aux marges des Etats, en confrontant dans la pratique quotidienne la diversité des approches, générera chez tous les acteurs impliqués cette ouverture de pensée et cet esprit communautaire sans lesquels une Europe unie ne pourra se construire.

Traduction : I. Bourgeois

\section{Indications bibliographiques}

-Behringer W., Clemens G., Geschichte des Saarlandes, Munich, 2009

-Bourgeois I. (dir.), Allemagne : compétitivité et dynamiques territoriales, Editions du CIRAC, 2007

-Bourgeois I., "Fédéralisme financier. Solidarité financière - l'expérience allemande », Regards sur l'économie allemande, $\mathrm{n}^{\circ}$ 102/2011

- Lasserre R., «La formation professionnelle en Allemagne. Dynamiques socio-économiques et capacités d'adaptation du système », Regards sur l'économie allemande, $n^{\circ}$ 113/2014

-Röhl K.-R., « «Fiscalité des PME : les effets pervers des réformes de 2008/09 », Regards sur l'économie allemande, $n^{\circ}$ 92/2009

-www.ceselorraine.eu/img_base/ged_v2/182/2011-cesel-rapport-allemagne.pdf

-www.institut-gr.lu

-www.saarland.de

- www.saarland.ihk.de

-www.saarland.de/dokumente/thema_SaarLorLux/Zukunftsbild_2020_-_frz_Internet-Fassung.pdf. 